\title{
LES MÉTAMORPHOSES PÂLISSANTES DANS LES TEXTES TRADUITS: ÉTUDE SUR LE JAUNE ET LE BLOND
}

\author{
Claudine Lécrivain
}

\author{
Universidad de Cádiz \\ Departamento de Filología Francesa e Inglesa \\ Avenida Gómez Ulla s/n \\ I I 07 I Cádiz \\ España \\ claudine.lecrivain@uca.es
}

\begin{abstract}
The following analysis is a reflection on the translations of the colour yellow and the shades of blonde found in a body of French and Spanish literary texts in an attempt to delimit the situations in which literal translations of these terms occur. Our analysis shall examine situations where translators propose distinct types of distancing from these literal translations through an application of their own colour filter. These interpretative strategies either manifest an intensification of realism or, on the contrary, an almost spontaneous solution to codified reading practices.
\end{abstract}

Keywords: colour, translation, Spanish literature, French literature

Afin de compléter une réflexion amorcée depuis l'année 200 I sur la traduction des termes de couleurs dans un corpus donné ${ }^{1}$, j’aborderai ici l'étude de JAUNE ainsi que celle de BLOND, inclus dans cette classe lexicale chromatique (classe qui présente environ 800 occurrences $^{2}$ dans le corpus de 9000 occurrences analysé).

\footnotetext{
${ }^{1}$ Voir à ce propos Estudios de Lengua y Literatura Francesas, № I 5 , Universidad de Cádiz, 2004: 7-21. Pages où est expliqué le choix de corpus, et où sont indiquées les titres des différents romans ainsi que les abréviations utilisées lors des citations. Le corpus étudié offre un ensemble de 9000 occurrences de couleurs, et présente un nombre similaire d'occurrences pour les textes originaux français et pour les textes originaux espagnols.

${ }^{2}$ Je n'ai pas retenu ici les références à différentes nuances telles que paille, ambre, safran, à cause de leur présence restreinte dans le corpus étudié. Et je n'ai pas inclus ici l'analyse de DORÉ (environ 270 occurrences), étant donné les limites du présent article.
} 